REVIEW ARTICLE

\title{
Targets identified from exercised heart: killing multiple birds
} with one stone

\author{
Hongyun Wang ${ }^{1,2}$, Yuling Xie ${ }^{1}$, Longfei Guan ${ }^{3}$, Kenneth Elkin ${ }^{4}$ and Junjie Xiao $\mathbb{D}^{1,2 凶}$
}

Cardiovascular diseases (CVDs) are a major cause of mortality worldwide, which are mainly driven by factors such as aging, sedentary lifestyle, and excess alcohol use. Exercise targets several molecules and protects hearts against many of these physiological and pathological stimuli. Accordingly, it is widely recognized as an effective therapeutic strategy for CVD. To investigate the molecular mechanism of exercise in cardiac protection, we identify and describe several crucial targets identified from exercised hearts. These targets include insulin-like growth factor 1 (IGF1)-phosphatidylinositol 3 phosphate kinase (PI3K)/ protein kinase B (AKT), transcription factor CCAAT/enhancer-binding protein $\beta$ (C/EBP $\beta$ ), cardiac microRNAs (miRNAs, miR-222 and miR-17-3p etc.), exosomal-miRNAs (miR-342, miR-29, etc.), Sirtuin 1 (SIRT1), and nuclear factor erythroid 2-related factor/ metallothioneins (Nrf2/Mts). Targets identified from exercised hearts can alleviate injury via multiple avenues, including: (1) promoting cardiomyocyte proliferation; (2) facilitating cardiomyocyte growth and physiologic hypertrophy; (3) elevating the antiapoptotic capacity of cardiomyocytes; (4) improving vascular endothelial function; (5) inhibiting pathological remodeling and fibrosis; (6) promoting extracellular vesicles (EVs) production and exosomal-molecules transfer. Exercise is one treatment ('stone'), which is cardioprotective via multiple avenues ('birds'), and is considered 'killing multiple birds with one stone' in this review. Further, we discuss the potential application of EV cargos in CVD treatment. We provide an outline of targets identified from the exercised heart and their mechanisms, as well as novel ideas for CVD treatment, which may provide novel direction for preclinical trials in cardiac rehabilitation.

npj Regenerative Medicine (2021)6:23; https://doi.org/10.1038/s41536-021-00128-0

Cardiovascular diseases (CVDs) have become a leading cause of death worldwide. In 2016, over three-quarters of death caused by CVD were heart attack and stroke, specifically. Several factors contribute to the increasing incidence of heart attack and stroke, including a sedentary lifestyle, cigarette smoking, and air pollution, etc. ${ }^{1-6}$. Thus, it is a vast and difficult challenge to prevent and treat CVDs. Exercise, however, benefits heart and reduces the risk of CVDs such as ischemic heart disease ${ }^{7}$, coronary heart disease $\mathrm{e}^{8-10}$ and heart failure ${ }^{11-14}$. Therefore, aerobic exercise is a primary recommendation for prevention of CVD and for clinical rehabilitation. Notably, not only rehabilitation exercise training, but also voluntary physical activity significantly improves cardiac function ${ }^{15,16}$. Physical capacity and exercise tolerance have even been demonstrated to be a prognostic factor for the patient outcomes in CVD ${ }^{14,17,18}$. Strenuous exercise or high-intensity endurance exercise may induce underlying deleterious influence on cardiac function ${ }^{19,20}$, which is not considered here. Considerations including low physical capacity and exercise intolerance are not yet in our discussion either. In this review, exercise refers to an appropriate intensity of aerobic exercise, including treadmill running, swimming and other forms of voluntary exercise.

We thoroughly review molecular targets identified from exercised hearts as well as the mechanisms of exercise-induced cardiac protection (Fig. 1). Specific targets identified from exercised hearts include insulin-like growth factor 1 (IGF1)phosphatidylinositol 3 phosphate kinase $(\mathrm{PI} 3 \mathrm{~K}) /$ protein kinase $\mathrm{B}$ (AKT) signaling, C/EBP $\beta$-CITED4 (transcription factor CCAAT/ enhancer-binding protein $\beta$-CREB-binding protein/p300- interacting transactivator with E/D-rich tail 4), cardiac miRNAs (miR-222, miR-17-3p, etc.), exosomal-microRNAs (miR-342, miR-29, etc.), SIRT1 (Sirtuin 1), and Nrf2/Mts (nuclear factor erythroid 2-related factor/metallothioneins). Briefly, exercise promotes cardiomyocyte proliferation, physiological hypertrophy, and antiapoptotic capacity. We also review related cell types (cardiac fibroblasts and endotheliocytes) in response to exercise. Given the potential value of extracellular vesicles (EVs) on CVDs treatment, we also discuss the benefits of exosomal-molecules in exercised hearts.

\section{EXERCISE REDUCES THE RISK FOR CVDS}

An effective intervention for CVD, exercise has been extensively studied. It protects the heart against cardiac disorders, such as heart failure, myocardial infarction (MI), hypertension, and insulin resistance ${ }^{17}$. (1) Exercise alleviates heart failure. An action trial with more than 3000 participants found that aerobic exercise causes a significant decrease in heart failure hospitalizations as well as decreased cardiovascular events ${ }^{21}$. (2) Exercise protects heart against $\mathrm{Ml}$, inhibiting the release of cardiac proinflammatory factors, which prevents pathologic changes ${ }^{22}$. Mechanistically, exercise augments the anti-apoptotic capacity and is a primary mechanism for its attenuating $\mathrm{Ml}$ development. For example, cardiac C/EBP $\beta$ reduction as well as an increase in miR-222 contributes to blocking Ml-induced cell apoptosis and, thus, pathological remodeling ${ }^{23}$. (3) Exercise intervention reduces blood pressure (BP) and attenuates hypertension ${ }^{24}$. The mechanism of

\footnotetext{
${ }^{1}$ Institute of Geriatrics (Shanghai University), Affiliated Nantong Hospital of Shanghai University (The Sixth People's Hospital of Nantong), School of Medicine, Shanghai University, Nantong, China. ${ }^{2}$ Cardiac Regeneration and Ageing Lab, Institute of Cardiovascular Sciences, Shanghai Engineering Research Center of Organ Repair, School of Life Science,

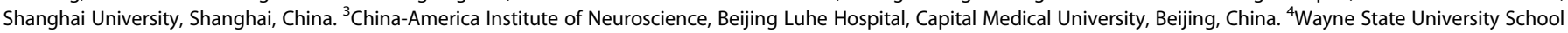
of Medicine, Detroit, MI, USA. ${ }^{\bowtie}$ email: junjiexiao@shu.edu.cn
} 


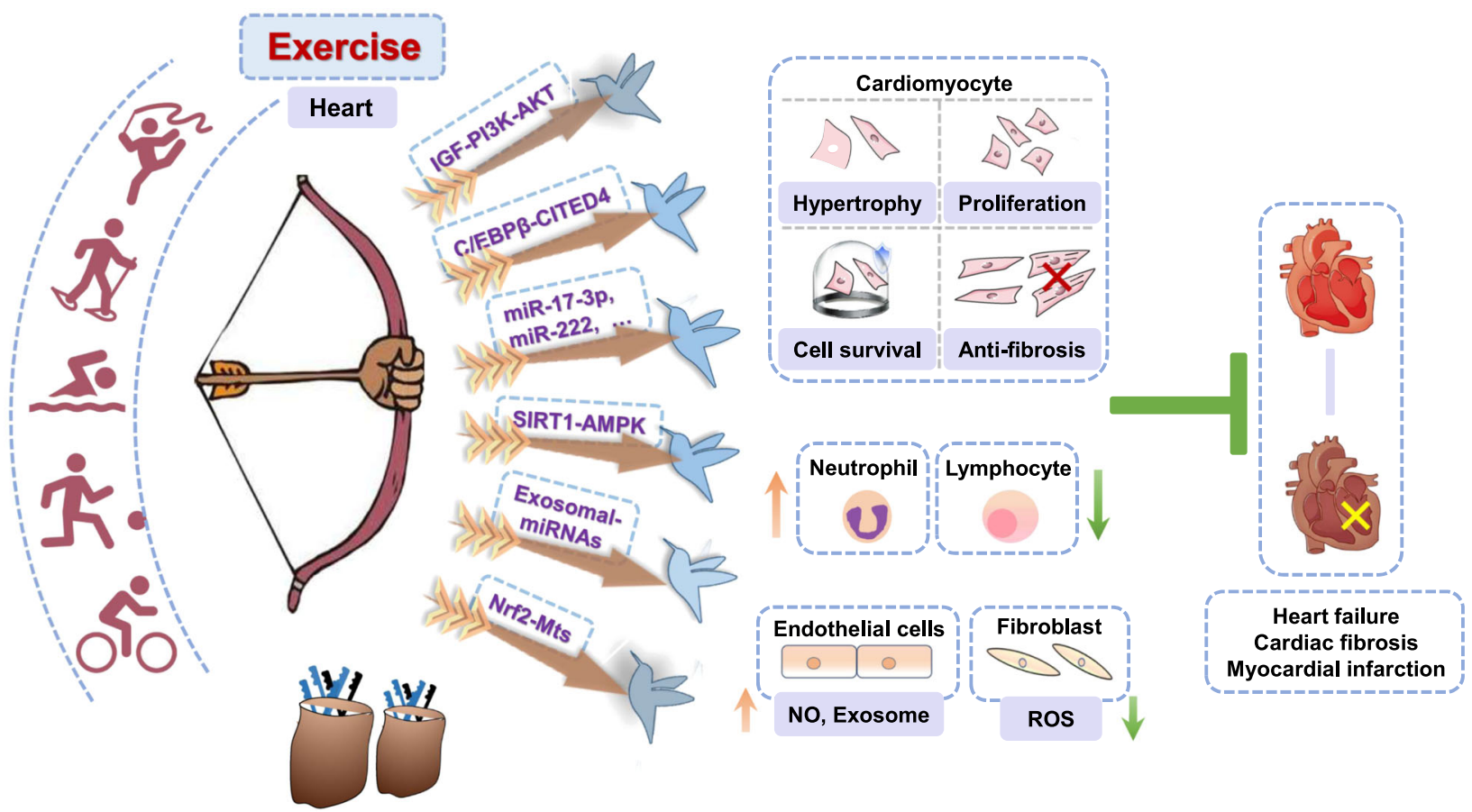

Fig. 1 Targets identified from exercised hearts protect against cardiac disorder via multiple avenues: killing multiple birds with one stone. Specific target identified from exercised heart (IGF1-PI3K/AKT signaling, C/EBP- $\beta$-CITED4, cardiac miRNAs (miR-222, miR-17-3p, etc.), exosomal-miRNAs (miR-342, miR-29, etc.), SIRT1 and Nrf2-Mts) was 'stone', multiple effects of exercise on hearts were 'birds'. Therefore, exercise protects hearts via 'killing multiple birds with one stone'.

exercise protecting BP is mainly associated with augmented endothelial functions ${ }^{25}$. As we discuss in part 3, exercise actives endothelial nitric oxide synthase (eNOS) which promotes nitric oxide (NO) production, and improves endothelial functions, mediating exercise-induced BP reduction. Additionally, oxidative stress in endothelial cells is also associated with exercise-induced endothelial protection ${ }^{26}$. (4) Exercise improves insulin resistance, reducing the risk for CVDs, as insulin resistance is a well-studied risk factor for CVDs according to a plethora of meta-analysis and systematic reviews. Clinically, insulin resistance is considered a better predictor of CVD events than fasting glucose levels ${ }^{27}$. Evidently, improving insulin sensitivity is beneficial to the cardiovascular health. Aerobic exercise can significantly improve insulin resistance and mitochondrial function, itself serving as an effective strategy for the treatment of metabolic syndrome ${ }^{28,29}$. Of note, the age, gender as well as exercise intensity should be considered when improving insulin resistance with exercise training. Different exercise intensity in different populations can produce different effects.

First, the effect of exercise on insulin resistance is associated with the intensity of exercise. In one study, low intensity physical exercise before each night shift did not affect glucose tolerance in rotating night shift workers ${ }^{30}$. High-intensity interval training, however, is found to significantly improve glucose homeostasis, functional capacity and body composition in healthy individuals ${ }^{31}$. A recent meta-analysis demonstrates that there is no difference in the glycemic and lipid level between resistance exercise and aerobic exercise ${ }^{32}$. Further, aerobic exercise alone or combined with resistance exercise provides similar effects on insulin sensitivity improvement in obese adolescents ${ }^{33}$. Aerobic exercise and resistance exercise combined with metformin are both effective in controlling glucose level. However, Walid found that resistance exercise is better than aerobic exercise when combined with metformin to treat the type 2 diabetes ${ }^{34}$.

Second, the protective effects of exercise vary with age and gender. For example, the young have been found to benefit more with respect to serum lipid and glucose homeostasis than the older after high-intensity exercise ${ }^{31}$. Resistance exercise-induced growth hormone and IGF1 molecular weight isoform are sex dependent ${ }^{35}$. Interestingly, Kaitlyn et al., report that the association between the exercise intensity and insulin sensitivity is also sex dependent, as it was observed that improvement of insulin sensitivity is associated with exercise intensity in obese men, but not obese women ${ }^{36}$.

\section{CELLULAR ADAPTIONS IN EXERCISED HEARTS}

In exercise-induced cardiac protection, many types of cells are physiologically altered. We examined four of these cells (cardiomyocytes, endothelial cells, cardiac fibroblasts, and immunocytes) and evaluate their changes following exercise (Fig. 2).

\section{Cardiomyocyte}

More energy consumption is required during exercise, leading to an increase in cardiac preload or afterload. Under this condition, cardiomyocytes tend to become physiologically hypertrophic (growth and proliferation) to match the increased demand. Adult cardiac physiological hypertrophy is characterized by an adaptive increase in cardiac mass as well as cardiomyocytes growth in length and width ${ }^{37}$ without collagen variation and maladaptive remodeling $^{38}$. Many signaling pathways contribute to exerciseinduced cardiac physiological hypertrophy, including IGF1-PI3KAKT-C/EBP $\beta$ and miRNAs, which are discussed in section 3.

\section{Vascular endothelial cells}

Moderate aerobic exercise improves vascular endothelial function via promotion of NO/vascular endothelial growth factor (VEGF) and of antioxidant enzyme. Additionally, the induction of superoxide dismutase (SOD) and peroxidase by exercise can inhibit reactive oxidative species (ROS) production in endotheliocytes ${ }^{39}$, and can also trigger endothelial cells to secret EVs and that 


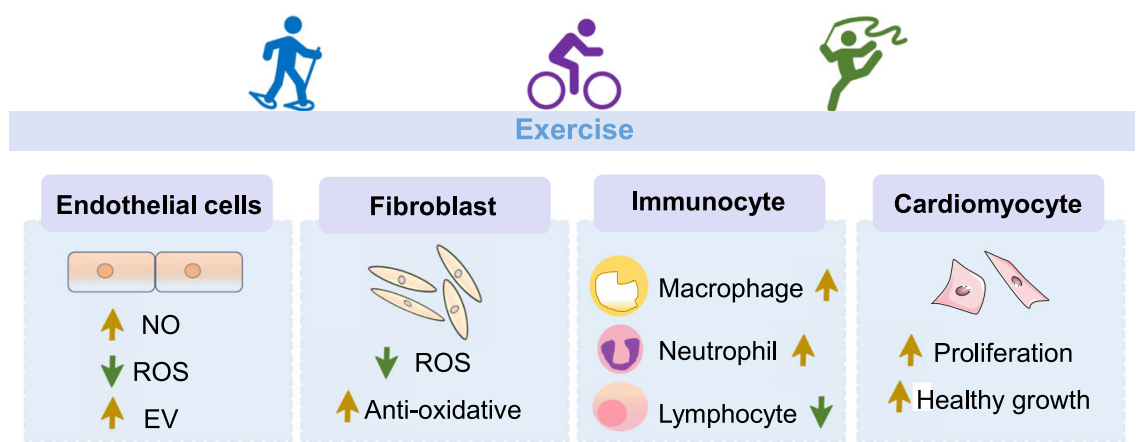

Fig. 2 Cellular adaptions in response to exercise. In endothelial cells, exercise inhibits ROS (reactive oxygen species) production and promotes NO (nitric oxide) production as well as EV (extracellular vesicles) secretion. Exercise also inhibits ROS and improves antioxidative capacity of cardiac fibroblasts. Besides, exercise induces a rapid increase in macrophage and neutrophil number, whereas decrease in lymphocyte number. All these adaptions directly or nondirectly contributes to exercise-induced cardiomyocyte proliferation and physiological hypertrophy.

influence cardiac function ${ }^{40}$. In the aging population, exercise is found to promote endothelial cell health by elevating CXC chemokine receptor (CXCR4)/janus kinase (JAK-2) signaling ${ }^{41}$.

\section{Cardiac fibroblasts}

Fibroblasts play critical roles in extracellular matrix remodeling and cardiac repair under certain stimuli, whose activation can interact with cardiomyocytes and maintain the cardiac microenvironment ${ }^{42}$. When activated, the fibroblast converts to a myoblast, contributing to cardiac repair or fibrosis ${ }^{43}$. Recent evidence demonstrates that exercise activated the nuclear factor erythroid 2-related factor (Nrf2) signaling in fibroblasts, subsequently elevating the expression of metallothioneins (Mts, including Mt1 and Mt2) and causing cardiomyocyte physiological hypertrophy ${ }^{44}$.

\section{Immunocyte}

Immunocytes are involved in exercise-induced cardiac protection. Clinical investigation indicates that circulating neutrophils and monocytes increase after exercise by promoting lymphocyte translocation to potential antigen sites in the heart. By contrast, exercise causes a significant reduction in lymphocytes number ${ }^{45}$.

\section{PIVOTAL TARGETS IDENTIFIED FROM EXERCISED HEART IGF1-PI3K-AKT signaling pathway}

The IGF1-PI3K-AKT pathway is extensively studied with respect to exercise-induced cardiomyocytes growth and hypertrophy ${ }^{46}$. IGF1 can be activated by exercise, and then binds to its receptors (IGF$1 \mathrm{R}$ ) on cardiomyocytes, causing intracellular PI3K/AKT pathway activation. During IGF-1R recycling, tumor susceptibility gene 101 (Tsg101) is found to interact with family-interacting protein 3 (FIP3), whose combination further increased recycling. Thus, Tsg101 is identified as a novel target in exercised heart, further increasing cardiomyocyte hypertrophy. Treadmill exercise training significantly promoted the expression of Tsg101 in mice and Tsg101-deficient mice counteracted exercise-induced cardiac physiological hypertrophy ${ }^{47}$. Additionally, Akt is a target of PI3K and has 3 isoforms (AKT1, AKT2, AKT3). Of these, AKT1 is required for exercise-induced cardiac physiological hypertrophy and growth $^{48}$. In cardiac-specific AKT1 transgenic mice, an $80 \%$ increase in AKT activity caused 2.2-fold increase in heart weight compared with that of control group ${ }^{49}$.

\section{C/EBP $\beta$}

$\mathrm{C} / \mathrm{EBP} \beta$, the transcription factor CCAAT/enhancer-binding protein $\beta$, is downstream of PI3K/Akt signaling, contributing to exercise- induced cardioprotection. Endurance exercise training can induce cardiomyocyte growth and proliferation, via AKT activation, and also suppresses C/EBP $\beta$ production. Inhibition of $C / E B P \beta$ in cardiomyocytes caused an increase in cell size and number, indicating that the C/EBP $\beta$ deficiency promoted the cardiomyocytes growth and proliferation ${ }^{50}$. Two axes are involved in C/EBP $\beta$ pathway in response to exercise: the C/EBP $\beta$-CITED4 axis and the C/EBPß-GATA4 axis (Fig. 3).

C/EBP $\beta$-CITED4 axis. Exercise-induced CITED4 upregulation can induce cardiomyocyte growth at basal condition ${ }^{50}$. CITED4 induces physiological hypertrophy ( 25\%-fold of increase in heart mass) without causing pathologic changes in male and female mice ${ }^{51}$, whose upregulation significantly promoted an increase in cell surface area, proliferation markers expression (Ki67 and EdU), and cell number ${ }^{52}$. Mechanistically, cardiac CITED4-induced hypertrophy is also associated with upregulation of neuregulin-1 $(\mathrm{NRG} 1)^{52}$.

C/EBPB-GATA4 axis. GATA4 is a key regulator of cardiac hypertrophy and cardiomyocyte viability ${ }^{53}$. Exercise training causes a significant increase in cardiac GATA4 expression in MI in mice, whose elevation is cardioprotective ${ }^{54}$. Mechanistically, GATA4 deficiency upregulates the expression of proapoptotic proteins (caspase12 and Bcl6), alters FGF1 (fibroblast growth factor 1) and EGFR expression, leading to a reduced hypertrophic response ${ }^{53}$.

\section{Cardiac microRNAs}

MicroRNAs, small noncoding RNA molecules ( 22 nucleotides), regulate gene expression in exercise-induced cardiomyocyte proliferation and growth. With RNA arrays or qRT-PCRs, several miRNAs have been identified from the exercised heart, such as miR-222, miR-17-3p, miR-21, and miR-124 $4^{55,56}$. Here, we review the roles and mechanisms of miR-222 and miR-17-3p in exerciseinduced cardiac physiological hypertrophy (Fig. 3).

MiR-222. MiR-222 was initially identified as a proproliferation factor in exercised mice models. To examine the microRNA profiling, microRNA arrays have been performed and revealed an increase in miR-222 content in exercised mice, which is consistent with that of the plasma of exercised heart failure patients. The elevation of miR-222 promotes cardiomyocyte growth and proliferation (Fig. 3). Interestingly, different targeted genes of miR-222 are found to exert different roles in cardiomyocyte. For example, miR-222 induces cardiomyocyte proliferation and growth via inhibition of Kip1 (p27) and homeodomain interacting protein kinase 1 (HIPK1). Genetic deletion of HIPK1 or p27 promotes 


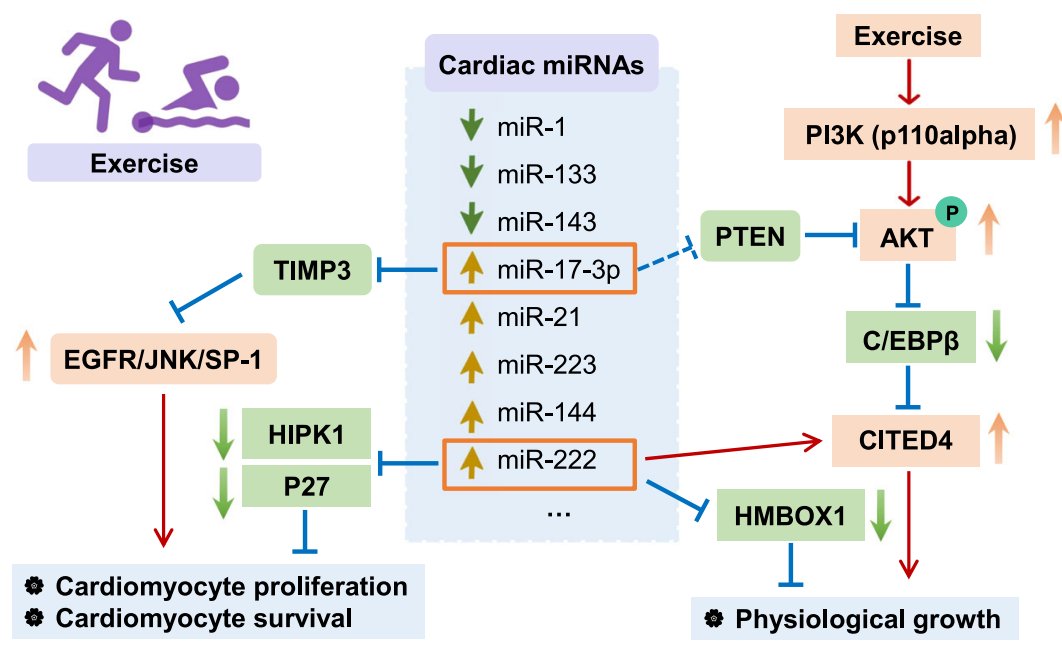

Fig. 3 MiRNAs in exercise-induced cardioprotection. The mechanism of PI3K/AKT, C/EBP- $\beta$-CITED4, and cardiac miRNAs (miR-222, miR-17-3p) in exercise-induced cardiomyocyte hypertrophy, proliferation, and survival.

cardiomyocyte physiological hypertrophy and proliferation, both mediators of exercise-induced cardiac protection. However, Homeobox Containing 1 (HMBOX1), another downstream target of miR-222, promotes cardiomyocyte growth and hypertrophy, but has little effect on the cardiomyocyte proliferation ${ }^{23}$.

MiR-17-3p. MiR-17-92 cluster is well studied and contributes to cardiomyocyte growth and proliferation ${ }^{57-59}$. The miR-17-92 cluster was initially reported in cancers and disorders, whose upregulation promotes cell proliferation ${ }^{60}$. Following this, investigators extensively examined the role of miR-17-92 cluster in hearts using cardiac-specifically transgene or knockout mice, finding miR-17-92-promoted cardiomyocyte proliferation in postnatal and adult hearts ${ }^{59}$. Notably, in passenger RNA of miR-17-92 cluster, miR-17-3p has been identified as a mediator for exerciseinduced cardiac growth and proliferation in mice models. In exercised hearts, miR-17-3p promotes cardiomyocyte proliferation, growth, and survival, via targeting of metallopeptidase inhibitor 3 (TIMP3), which activates epidermal growth factor (EGF) receptor (EGFR), and c-Jun $\mathrm{NH}(2)$-terminal kinase (JNK)/SP-1 ${ }^{57}$. Lastly, miR$17-3 p$ indirectly regulates the phosphate and tension homology on chromosome ten (PTEN)/ protein kinase B (AKT) pathway, promoting cardiomyocyte cell survival.

\section{Exosomal-microRNAs}

Small EVs, or exosomes, are lipid bilayer vesicles with a diameter of $40-200 \mathrm{~nm}$, secreted by most types of cells and existing in biological fluid such as bloods, urine, and saliva. Exosomes play important roles in cell-to-cell crosstalk, using their molecular cargo to communicate ${ }^{61-63}$. They are considered as a potential therapeutic strategy due to their immunologic inertia and stabilization $^{61}$. MiRNAs in the exosomes play important roles in exerciseinduced cardiac protection; known exosomes include miR-342-5p, miR-455, miR-320, miR-29, and miR-126. The mechanism of these exosomal-miRNAs in exercise-induced cardiac protection is shown in Fig. 4. Here in this section, we review function of exosomal-miR$342-5 p$ in exercise-induced cardioprotection.

Exosomal-miR-342-5p. MiR-342 is a mediator of exercise-induced cardiac protection. MiRNA sequencing reveals endothelial cellderived exosomal-miR-342-5P undergoes a robust increase in exercised athletes and swam rats. Exercise-induced exosomal-miR342-5P elevation improves the anti-apoptosis capacity of cardiomyocyte, mediated through inhibition of caspase 9 and cardiac cJun N-terminal kinase (JNK2) ${ }^{64}$ (Fig. 5). Also, a recent study in

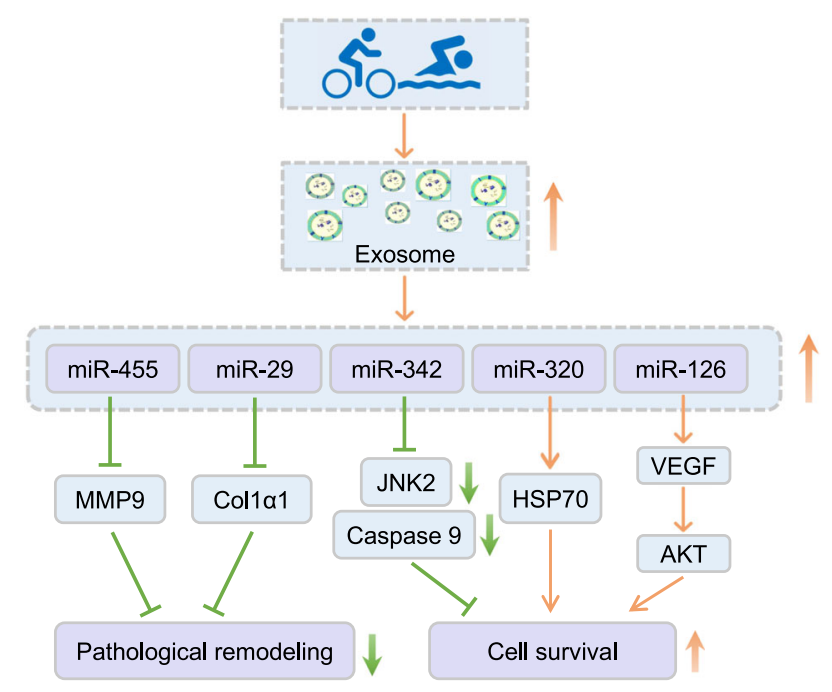

Fig. 4 Exosomal-miRNAs identified from exercised hearts. Exercise promotes EVs and exosomal-miRNAs secretion. This figure shows the mechanism of exosomal-miR-455, miR-29, miR-342, miR-320, and miR-126 in exercise-induced cardiac protection.

exercised healthy subjects showed that exercise dramatically induced 12 exosomal-miRNAs (miR-1-3p, -208a-3p, -486-5p, -23a$3 p,-23 b-3 p,-451 a,-16-5 p, 378 a-5 p,-126-3 p,-150-5 p,-222-3 p$, and $-186-5 p)$ increased in the exosome fraction ${ }^{65}$.

\section{SIRT1 signaling}

Exercise significantly increases SIRT1 expression ${ }^{66}$. SIRT1, one of the members of the sirtuin protein family, is an enzyme responsible for protein deacetylation, endoplasmic reticulum (ER) stress, oxidative stress, and energy metabolism. Increasing studies demonstrate that SIRT1 is involved in exercise-induced cardiac protection by alleviating adverse cardiac remodeling ${ }^{67}$ and reducing cell apoptosis ${ }^{68}$. Mechanistically, SIRT1 plays protective roles via (1) peroxisome proliferator-activated receptor- $\gamma$ coactivator 1a (PGC-1a) activation, (2) eukaryotic initiation factor (elF2a) pathway inactivation, and (3) forkhead boxo1 (FOXO1) reduction. First, SIRT1/PGC-1a signaling pathway activation can promote cell survival ${ }^{69}$. Exercise training upregulates SIRT1/PGC-1a/AMPK (AMP-activated protein kinase) and prolongs cell longevity ${ }^{70}$. 


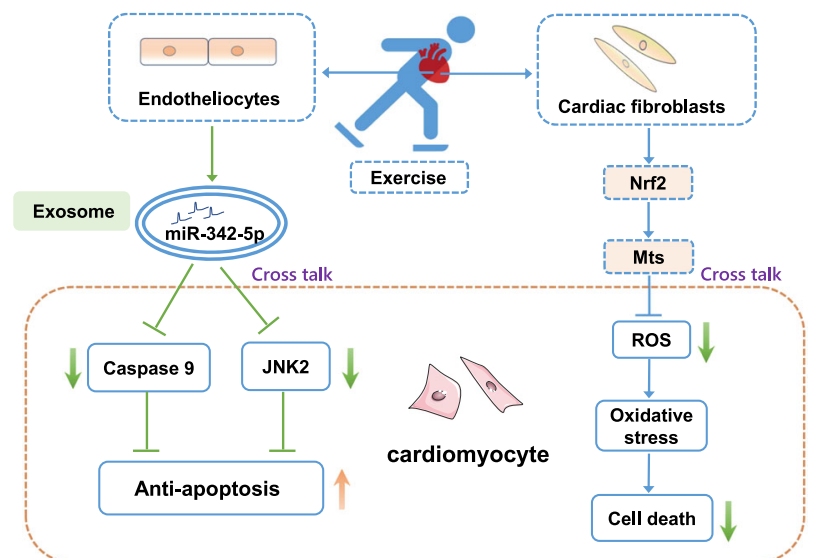

Fig. 5 Crosstalk between noncardiomyocyte and cardiomyocytes in exercise-induced cardiac protection. Exercise promotes the expression of exosomal-nuclear factor-erythroid 2 related factor 2 (Nrf2)/metallothioneins (Mts) in cardiac fibroblasts, inhibiting cardiomyocyte reactive oxidative species (ROS) production and cell apoptosis. Endotheliocytes produce EVs (containing miR-342-5p) and deliver to cardiomyocyte, leading to an increase in the antiapoptosis capacity via inhibiting caspase 9 as well as c-Jun Nterminal kinase (JNK2).

Second, SIRT1 can attenuate ER stress-induced heart injury and cell apoptosis by interacting with elF2 $a$ and inducing deacetylation of the lysine site (K141 and K143), which leads to protein kinase R-like endoplasmic reticulum kinase (PERK)/ elF2a pathway inactivation ${ }^{71}$. Third, SIRT pathway activation regulates the deacetylation of FOXO1 and alleviates oxidative stress ${ }^{72}$.

\section{Nrf2-dependent pathway}

Nrf2, a transcriptional regulator, has a central rols in antioxidative response by regulating more than 200 antioxidant genes such as $\mathrm{NAD}(\mathrm{P}) \mathrm{H}$ quinone oxidoreductase 1 (NQO1), glutathione-Stransferases (GSTs) and hemeoxygenase $1(\mathrm{HO}-1)^{73}$. When activated by stress and, notably, exercise, Nrf2 can translocate from cytoplasm to nucleus and trigger cytoprotective gene expression, thereby improving cardiac antioxidant capacity ${ }^{74}$ (Fig. 5). Mechanistically, ROS production promotes Nrf2 translocation and activation, leading to nuclear Nrf2 accumulation in the exercised mice heart. Consistently, one study reveals that exercise activates Nrf2 signaling in the exercised young person, thereby elevating the expression of antioxidant genes such as $\mathrm{HO}-1, \mathrm{NAD}(\mathrm{P}) \mathrm{H}^{75}$. The content of Nrf2 activation is associated with the intensity of exercise, whose activation has different adaptions in different exercise protocols.

\section{THE MECHANISM OF EXERCISE-INDUCED CARDIAC PROTECTION}

Exercise induces several cellular adaptions, including proliferation and physiological hypertrophy of cardiomyocytes, antioxidation of myoblasts, production of endothelial NO, and a number changes in immunocytes. In this section, we review the mechanisms by which these adaptations are cardioprotective (Fig. 6).

\section{Exercise leads to cardiomyocyte growth and physiological hypertrophy}

Myocardial hypertrophy is an adaptive response to growth factors and increased workload, and includes physiological and pathological hypertrophy. Studies exploring the effects of exercise on cardiac size and left ventricular function have demonstrated that exercise caused an increase in cardiac size and improvement in left ventricular diastolic function, which is physiological, rather than pathological, hypertrophy ${ }^{76}$. Physiological hypertrophy is a typical manifestation of the cardioprotective effects of exercise, quite different from pathologic hypertrophy ${ }^{77,78}$. The serum level of natriuretic peptides ( $\mathrm{N}$-terminal proatrial and probrain natriuretic peptide) are biomarkers, which allow differentiation between physiological and pathologic cardiac hypertrophy ${ }^{79}$. We propose that the molecular changes identified from exercise-induced physiological may be potential targets for prevention and treatment of CVDs. Mechanistically, the decreased fatty acid oxidation and increased glucose utilization are important molecular adaptations in response to increased workload ${ }^{80}$. We review important molecular alterations identified from exercise-induced physiological hypertrophic heart. Animal and clinical studies demonstrate that exercise training significantly improved physiological cardiomyocytes growth via activating the IGF1-PI3K/AKT pathway ${ }^{81}$, whose activation also improved the antiapoptotic capacity of cardiomyocytes. Exerciseinduced IGF1-PI3K/AKT pathway activation is a crucial regulator of pressure overload-induced heart dysfunction ${ }^{82}$ and $\mathrm{MI}^{83}$. The activation of the IGF1-PI3K-AKT signaling pathway mediates exercise-induced cardiac physiological hypertrophy, whose activation may be a potential target for CVD therapy.

As one of the downstream targets of PI3K/AKT, C/EBP $\beta$-CITED4 is also important in exercise-induced cardiac healthy growth. For example, a reduction in C/EBP $\beta$ mitigates the pressure overloadinduced heart failure in mice ${ }^{50,84}$. In exercised mice, C/EBP $\beta$ deficiency inhibits NF-KB activation, thereby protecting cardiomyocytes from pathological hypertrophy ${ }^{85}$.

Functionally, physical exercise-induced physiological cardiac hypertrophy may compensate for the pathological myocardial remodeling such as $\mathrm{MI}^{67,86-88}$. A population study demonstrates that long-term endurance exercise caused cardiac remodeling not only in the left ventricle ${ }^{87}$, but also in the right ${ }^{89,90}$. Preclinical experiments demonstrates that voluntary exercise significantly promoted cardiac $\beta_{3}$-Ars ( $\beta 3$-adrenergic receptor) expression, which is cardioprotective against ischemia reperfusion injury (IR/ I). Physical exercise-induced cardiac $\beta_{3}$-Ars elevation activates eNOS, which eventually stimulated NO production ${ }^{91}$. The $\beta_{3}$-Ars$\mathrm{NO}$ axis is a novel mechanism involves in exercise-induced cardiac healthy remodeling and protection.

\section{Exercise promotes cardiomyocytes proliferation}

Mammalian cardiomyocytes have an extremely low proliferation rate $^{92}$, which gradually decreases from 1 to $0.3 \%$ at the age above 70 years in human. The renewal of cardiomyocytes is no more than $50 \%$ over a lifetime ${ }^{93,94}$. Exercise promotes the capacity of cardiac proliferation in adult mice. In one study, two months of treadmill running increases the generation of cardiomyocytes ${ }^{95}$. Recently, Ana Vujic et al. used the multi-isotope imaging mass spectrometry (MIMS) to study cardiomyocyte proliferation and found $\sim 4.6$ folds of increase in adult exercised mice ${ }^{95}$.

Several molecules are involved in exercise-induced cardiomyocyte proliferation, such as C/EBP $\beta$, miRs, and their downstream targets. Decreased C/EBP $\beta$, or increased miR-222, both inhibit cardiac disorder via promoting proliferation and growth. C/EBP $\beta$ was the first transcriptional factor identified from adult exercised hearts, whose reduction has been associated with exercise-induced cardiomyocyte proliferation ${ }^{50}$. CITED4, which is downstream of C/ $E B P \beta$, contributed to exercise-induced cardiac protection. Exercise elevates GATA4, CITED4, and reduces C/EBP $\beta$ in MI mice, thereby protecting against cardiac disorder. Compared with the sham group, the Ml mice exhibites a higher expression of C/EBP $\beta$. Disruption of C/EBP expression in the adult heart inhibits MIinduced neutrophil infiltration and improved cardiac function ${ }^{51,96}$. CITED4 can attenuate cardiomyocyte apoptosis and autophagy flux after IR/I injury by activating the mTORC1 pathway ${ }^{51,97}$. Additionally, microRNAs, such as miR-222, miR-342, miR-17 are involved in exercise-induced cardiac proliferation. Cardiac miR-222 targets p27, HIPK1/2 and Hmbox1 and alleviates MI-induced injury. 


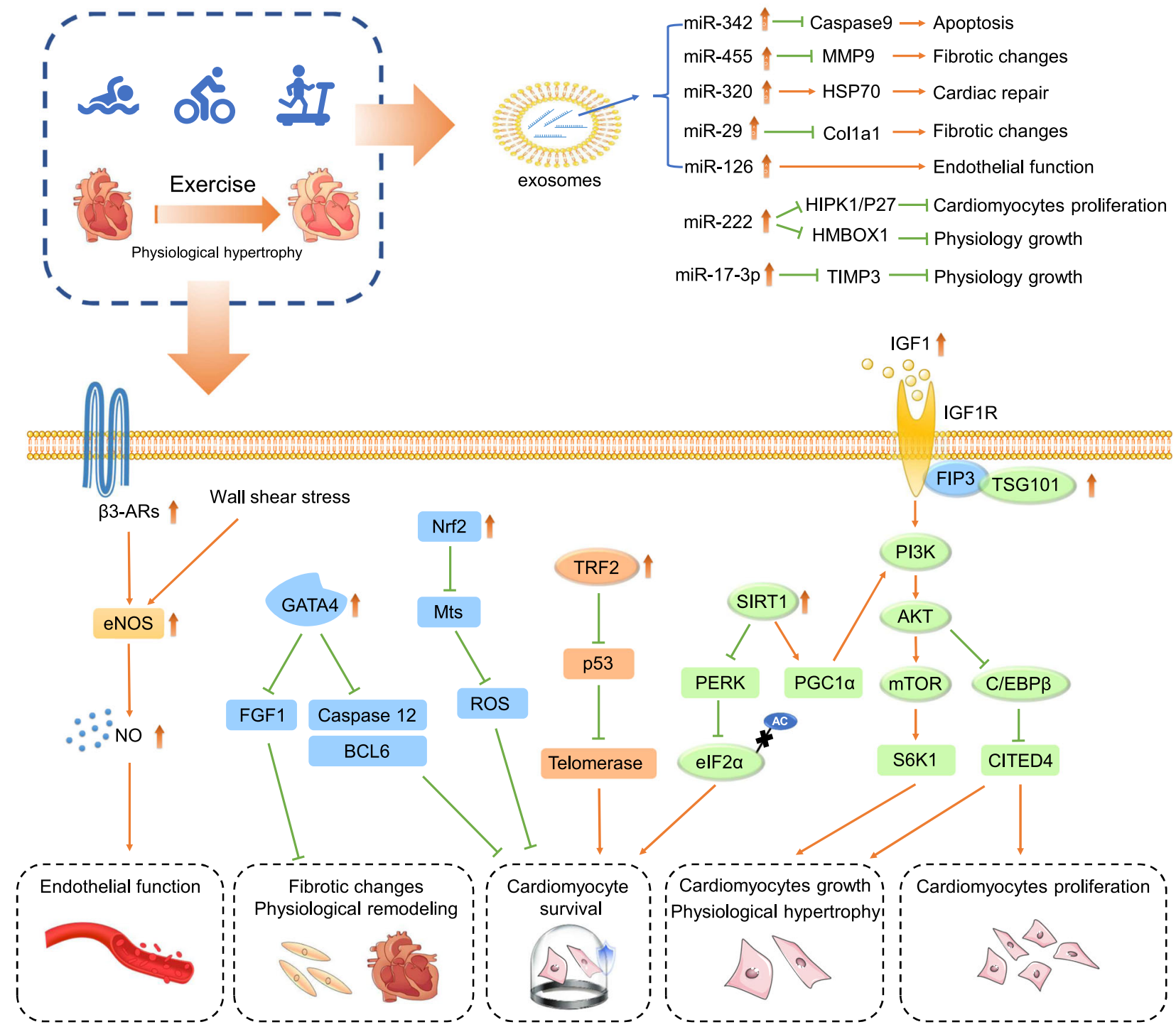

Fig. 6 The targets identified from exercised hearts and their mechanism: killing multiple birds with one stone. In this section, we give an insight on exercise-induced cardioprotection, which includes (1) promoting cardiomyocytes proliferation; (2) facilitating cardiomyocytes growth and physiologic hypertrophy; (3) elevating the anti-apoptosis capacity of cardiomyocyte; (4) promoting vascular endothelial function; (5) inhibiting pathological remodeling and fibrosis; (6) promoting EVs production and exosomal-molecular transfer to cardiomyocytes.

Interestingly, exercise-induced cardiomyocyte proliferation may vary with age. To investigate this issue, different ages of rats (juvenile, adolescent and adult) are used in a recent study that underwent treadmill running for 4 weeks. Cell number analysis shows that the juvenile group has a significant increase in cardiomyocyte number (36\%), higher than the adolescent and adult group. Exercise-induced cardiomyocyte proliferation is associated with the period of life ${ }^{98}$. Investigations in zebrafish also indicated that exercise training can promote cardiomyocyte proliferation ${ }^{99}$. To build the physiological hypertrophy model, adult zebrafish $(\sim 2.5 \mathrm{~cm}$ in body length) swam for sum up to 13 days, inducing an increase in cardiomyocytes proliferation ${ }^{100}$. As cardiomyocyte loss is a primary cause of CVD, such as in heart failure and myocardial infarction ${ }^{92,101-103}$, exercise-induced cardiomyocyte proliferation may be a potential preventative and therapeutic strategy for CVD.

Notably, the cell to cell crosstalk between cardiomyocytes and noncardiomyocytes plays important roles in cardiomyocyte proliferation $^{42}$. Myofibroblasts, endotheliocyte and adipocytes can secret growth factors and cytokines and promote cardiomyocyte proliferation. In large animals, the isolated sinoatrial node cells (SANCs) from guinea pig heart can promote the generation of cardiomyocytes ${ }^{104}$, and may be an area of future study.

\section{Exercise improves the anti-apoptosis capacity of cardiomyocytes}

Cardiomyocyte apoptosis is another cause of $\mathrm{CVD}^{105,106}$, which can be ameliorated by exercise intervention. In the treadmill exercise model, the apoptosis index ( $\mathrm{Bax} / \mathrm{Bcl} 2$, cleaved caspase 3/caspase 3) decreases after two months of running ${ }^{107}$. PI3K/AKT signaling inhibits cell apoptosis and promotes cardiomyocyte survival in exercise-induced cardiac function improvement in $\mathrm{Ml}$ mice. Additionally, exercise inhibits TGF $\beta 1$ activation and leucocytes activation and migration, leading to an increase in anti-apoptotic capacity ${ }^{86}$. miR-222 inhibits TGF $\beta 1$ pathway (JNK, TGF- $\beta$ receptor), which is cardioprotective against TAC or angiotensin Il-induced remodeling and cardiac fibrosis ${ }^{108}$.

In the past decade, the association between telomerase activity and exercise training has received widespread attention. Voluntary running enhances the cardiac telomerase activity to $\sim 2$-fold of control, and caused an increase in TRF2 (telomere repeat binding 
factor 2) expression and $\mathrm{p} 53$ reduction ${ }^{109}$. Exercise also promotes the expression of telomere-protective genes and extends telomere length by activating the p38 MAPK pathway in myocardial cells $^{110}$. Exercise attenuated stress-induced telomerase activity reduction and telomerase length shortening, which has been shown to prevent coronary heart disease ${ }^{111}$.

Furthermore, exercise diminishes cardiomyocyte apoptosis in an indirect way, including increased autophagy content and mitochondrial function. In doxorubicin-treated mice, endurance running exercise reduced dox-induced cell apoptosis by increasing the autophagy content containing increased LC3II and decreased p62, as well as mitochondrial autophagy ${ }^{112}$.

\section{Exercise improves vascular endothelial function}

Endothelial cells play important roles in vascular homeostasis, whose dysfunction contributes to the pathological process of CVD. Aerobic exercise promotes NO production, balancing endotheliumdependent vascular homeostasis and regulating endothelial dysfunction in patients with heart failure ${ }^{113}$ and hypertension ${ }^{114}$. NO is produced by three kinds of nitric oxide synthase (NOS) enzymes: eNOS, neuronal nitric NOS (nNOS), and inducible NOS (iNOS) $)^{115}$. The activation of eNOS is associated with the endothelial function. Exercise training improves local blood flow by these means ${ }^{116}$ and coronary arteries vasodilation as well ${ }^{117,118}$. However, the mechanism of eNOS or NO upregulation after exercise is unknown. A recent study demonstrates that the change of wall shear stress (WSS) induced by exercise may contribute to the increase in NO production ${ }^{119}$.

MicroRNAs regulate endothelial function in exercised hearts and become a potential diagnostic biomarker for physical capacity ${ }^{120}$. Experimental evidence has revealed that exercise has a profound effect on the circulating miRNA profile. For example, exercise causes a significant increase in cardiac miR-492 expression in aortic endothelial cells and improves endothelial cell function ${ }^{121}$. Additionally, miR-126 induced by aerobic exercises inhibits the production of endothelium-derived factors such as NO and endothelin, eventually leading to further improvement in endothelial function ${ }^{122}$.

Of note, exercise-induced cardioprotective role of circulating microRNAs is not only through improving the endothelial function, but also in directly attenuating the progression of heart diseases. Specific exercise interventions such as Chinese Tai Chi can improve the life quality and block the progression of coronary heart disease (CHD) via inhibiting miR-24 and miR-155, whose level is lower in the serum of Tai Chi exercised CHD patients than that of sedentary ones ${ }^{123}$.

\section{Exercise inhibits fibroblast switch and cardiac fibrosis}

Cardiac fibroblasts, or myofibroblasts, play integral roles in extracellular matrix degradation, repair, inflammation, and cardiac disease development ${ }^{42}$. Cardiac fibroblasts converting to myofibroblasts can lead to collagen deposition and tissue fibrosis, ultimately leading to pathological events ${ }^{124}$. Notably, cardiac fibroblasts are involved in exercise-induced cardiac growth due to their communication with cardiomyocytes $^{78}$. To evaluate the effect of cardiac fibroblasts on exercise-induced cardiac growth, RNA sequencing is performed in exercised or diseased C57BL/6 mice. The transcriptional analysis demonstrates that antioxidant gene Nrf2 and metallothioneins ( $\mathrm{Mt}$, including Mt1 and Mt2) increase in exercised hearts, while they decrease in $\mathrm{Ml}$ and TAC hearts. Furthermore, Mt1 and Mt2 deficiency exacerbates cardiac dysfunction in mice with MI. Mechanistically, the elevation of Mts in cardiac fibroblasts can transfer to cardiomyocytes, eventually inhibiting cardiomyocyte apoptosis ${ }^{44}$.

MiRNAs, such as miR-29, are a crucial mediator for exerciseinduced physiological remodeling, leading to resistance to pathological myocardial remodeling ${ }^{125}$. MiR-29 significantly attenuates cardiac collagen deposition after MI injury via inhibition of cardiac collagen type I alpha 1 (CollA1) ${ }^{126}$. Also, exercise attenuates cardiac fibrosis via activating the SIRT1/PGC1a/PI3K/Akt pathway ${ }^{67}$.

Furthermore, running can protect the heart against cardiac fibrosis in female ovariectomized mice by reducing oxidative stress through 8-hydroxy-2'-deoxyguanosine, matrix metalloprotein 2 (MMP2) and collagen I/III reduction ${ }^{127}$. Of note, a low intensity of exercise still promotes the antioxidative activity of heart and improves the diastolic function of left ventricle, which is different from the function on right-ventricle ${ }^{128}$.

\section{Exercise promotes EVs production}

Considered cargo in the body, EVs contain multiple molecule types including $m R N A$, microRNA, DNA, and proteins, mediating a wide variety of physiological and pathological processes ${ }^{129,130}$. Exercise induces the production of extracellular vesicles both in humans and experimental animals ${ }^{131-133}$. Of note, EVs are secreted into system circulation by almost all kinds of cells, including cardiomyocytes, endothelial cells, and platelets ${ }^{40}$. EVs deliver miRNA and proteins from endothelial cells or fibroblasts to cardiomyocyte cells in response to exercise, thereby having some role in regulation of cardiac function. In a diabetic heart model, the exercised heart tissues, as well as serum, are collected for isolation and analysis of EVs. A microRNA profile analysis showed that exercise significantly elevated miR-29b and miR455 with respect to the sedentary group, inhibiting the expression of matrix metalloprotein 9 (MMP9) and cardiac remodeling ${ }^{134}$. Also, miR320 in the EVs is potent in cardiac repair, whose presence improves hyperglycemia-induced cardiac injury via HSP20 pathway activation $^{135}$. Exosomal-miR-126, produced by endothelial cells, increases after exercise ${ }^{136}$. EVs derived from these endothelial progenitor cells can promote vascular angiogenesis and protect heart function by delivering miR-126 in exercised hearts ${ }^{137}$. VEGF, the potential downstream target of miR-126, promotes the activation of AKT and ERK pathways, thereby improving cell survival ${ }^{138}$.

Additionally, EVs content differs greatly with and without exercise. Quantitative proteomic analysis is performed in EVs from the serum of exercised and rested human to figure out the protein cargo composition. Results showes that more than 300 proteins are transferred into system circulation ${ }^{139}$. The proteins enclosed in EVs are associated with several processes such as glycolysis (Glucose-6-phosphate 1-dehydrogenase elevation), which is activated to meet the energy requirements of exercise. As natural EVs and its contents play central roles in crosstalk between noncardiomyocyte and cardiomyocyte, EVs emerge as a potential therapeutic strategy for CVD.

\section{Exercise reduces metabolic disorders and transcriptional changes}

With economic development, the burden of cardiometabolic risk rapidly expands and attracts much attention. Cardiometabolic disease-induced mortality including insulin resistance, type 2 diabetes mellitus, and obesity, are increasing. Wang et al., reportes a significant increase in cardiometabolic risk among Chinese adults in a recent study ${ }^{140}$. Obesity and elevated dyslipidemia in younger populations implies an increasing potential of cardiometabolic diseases. Aerobic exercise, as we have seen, can ameliorate these effects, improving insulin resistance ${ }^{28}$, glucose level ${ }^{141}$ and overweight ${ }^{142}$ and reduce the cardiometabolic risk ${ }^{143,144}$. Exercise-induced transcriptional changes are important to reducing insulin resistance, partially accomplished through the transcription of insulin resistance-related genes and mitochondrial DNA (mtDNA) (Fig. 7).

Studies exploring transcriptional changes induced by exercise in skeletal muscle have shown that genes related to muscle growth and antiatrophy are significantly remodeled. Among these, ANGPTL4 (angiopoietin like 4) is regulated by exercise, whose gene expression is associated with DNA methylation ${ }^{145}$. Hu et al. analyzes gene profiles and transcriptional changes induced by 


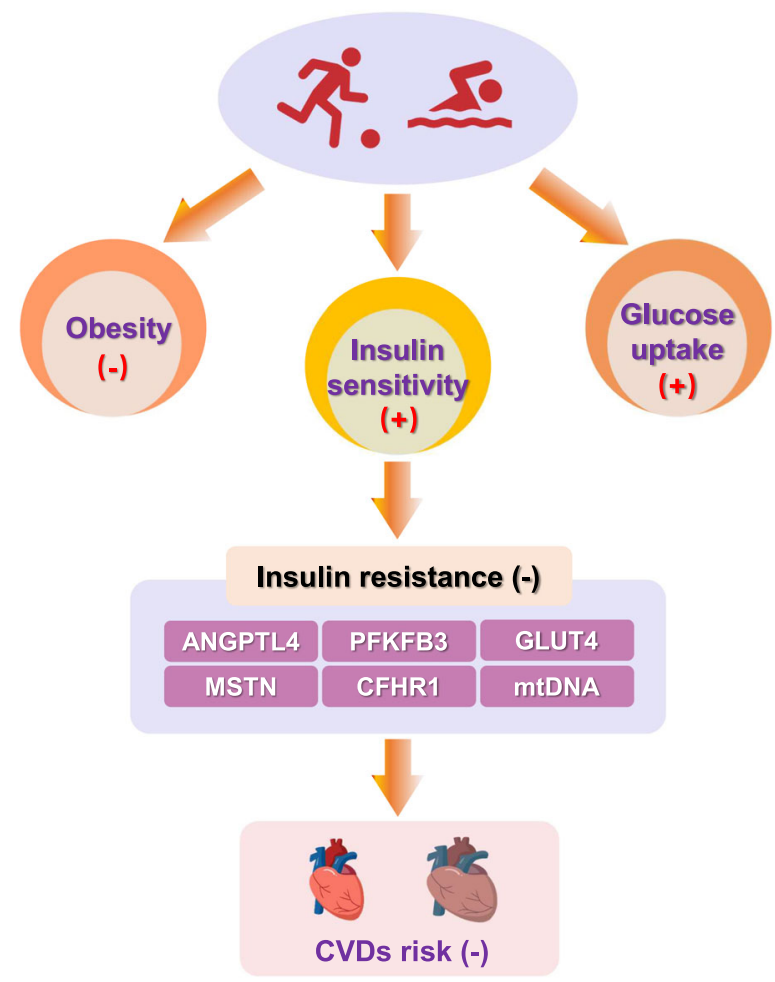

Fig. 7 The effects of exercise on insulin resistance and transcriptional changes. Exercise improves insulin resistance via reducing obesity, improving insulin sensitivity, and glucose uptake.

exercise in resistant patients and healthy controls with exercise training. Differentially expressed genes are analyzed and reveals that alteration of insulin resistance-related genes, such as MSTN (myostatin), CFHR1 (the complement factor $\mathrm{H}$ related 1), PFKFB3 (6phosphofructi-2-kinase/fructose-2,6-biphosphatase) were reversed by exercise ${ }^{146}$. In addition, transcriptional changes of genes associated with glucose metabolism are investigated during exercise, ultimately demonstrating improved glucose uptake and oxidation by increasing glucose transporter 4 (Glut4) transcription in a AMPKa-independent manner ${ }^{147}$.

Mitochondrial DNA (mtDNA) is also associated with insulin sensitivity. Tomas et al. report that insulin resistance is associated with decreased mtDNA content in adolescents ${ }^{148}$. Additionally, mtDNA copy number is considered a biomarker of glucose homeostasis and insulin sensitivity in nondiabetic woman ${ }^{149}$. Cytochrome b (Cytb) is one of these genes encoded by mtDNA, and is decreased in type 2 diabetic mice when compared to control. The dyslipidemia-induced Cytb mRNA reduction, in one study, is rescued by aerobic exercise ${ }^{150}$.

\section{CONCLUSIONS AND PERSPECTIVES}

In this review, we examine the cardiac benefits of exercise and summarize the key factors, and their mechanisms, in exerciseinduced cardiac protection, including IGF1-PI3K/AKT signaling, C/EBP- $\beta$-CITED4, cardiac miRNAs (miR-222, miR-17-3p, etc.), exosomal-miRNAs (miR-342, miR-29, etc.), SIRT1 and Nrf2-Mts. Furthermore, we review the role of noncardiomyocytes in exercise-induced cardioprotection, including cardiac fibroblasts and endothelial cells. These cardioprotective mechanisms include the following: (1) PI3K/AKT activation which reduces C/EBP- $\beta$ expression and causes CITED4 and GATA upregulation after exercise training. This ultimately leads to an increase in antiapoptotic activity and enhancement of physiological hypertrophy. (2) Increased miR-222 induces physiological hypertrophy of cardiomyocytes via inhibiting HMBOX1 expression. MiR222 mimic supplement can promote cardiomyocyte proliferation via suppression of HIPK1/p27. (3) miR-17-3p targets TIMP3, activates EGFR/JNK/SP-1, and regulates PTEN/AKT pathway, leading to cardiomyocyte cell survival. (4) Exosomal-miR-342, produced by endotheliocytes and delivered to cardiomyocytes, can attenuate cardiomyocyte apoptosis via inhibition of caspase 9 and JNK2. (5) SIRT1 signaling promotes the antiapoptotic capacity of cardiomyocytes via inhibition of p53, oxidative stress, and elF2a acetylation. (6) Nrf2/Mts expression, in fibroblasts, induces Mts translocation to cardiomyocytes, thereby suppressing oxidative stress in cardiomyocytes. In conclusion, targets identified from exercised heart may have therapeutic potential in cardioprotection in CVDs, 'killing multiple birds with one stone' (Fig. 6).

\section{DATA AVAILABILITY}

The data that support the findings of this study are available from the corresponding author upon reasonable request.

Received: 1 July 2020; Accepted: 26 February 2021; Published online: 09 April 2021

\section{REFERENCES}

1. Lee, I., Kim, S. \& Kang, H. Lifestyle risk factors and all-cause and cardiovascular disease mortality: data from the Korean Longitudinal Study of Aging. Int. J. Environ. Res. Public Health https://doi.org/10.3390/ijerph16173040 (2019).

2. Pope, C. A. 3rd et al. Cardiovascular mortality and long-term exposure to particulate air pollution: epidemiological evidence of general pathophysiological pathways of disease. Circulation 109, 71-77 (2004).

3. Nansseu, J. R. et al. Prevalence of major cardiovascular disease risk factors among a group of sub-Saharan African young adults: a population-based crosssectional study in Yaounde, Cameroon. BMJ Open 9, e029858 (2019).

4. Hubert, H. B., Feinleib, M., McNamara, P. M. \& Castelli, W. P. Obesity as an independent risk factor for cardiovascular disease: a 26-year follow-up of participants in the Framingham Heart Study. Circulation 67, 968-977 (1983).

5. Stamler, J., Vaccaro, O., Neaton, J. D. \& Wentworth, D. Diabetes, other risk factors, and 12-yr cardiovascular mortality for men screened in the Multiple Risk Factor Intervention Trial. Diabetes Care 16, 434-444 (1993).

6. Kannel, W. B. \& McGee, D. L. Diabetes and cardiovascular disease. The Framingham study. JAMA 241, 2035-2038 (1979).

7. Kyu, H. H. et al. Physical activity and risk of breast cancer, colon cancer, diabetes, ischemic heart disease, and ischemic stroke events: systematic review and doseresponse meta-analysis for the Global Burden of Disease Study 2013. BMJ 354, i3857 (2016).

8. Wang, L., Ai, D. \& Zhang, N. Exercise benefits coronary heart disease. Adv. Exp. Med. Biol. 1000, 3-7 (2017).

9. Moraes-Silva, I. C., Rodrigues, B., Coelho-Junior, H. J., Feriani, D. J. \& Irigoyen, M. C. Myocardial infarction and exercise training: evidence from basic science. Adv. Exp. Med. Biol. 999, 139-153 (2017).

10. Marschner, R. A. et al. Short-term exercise training improves cardiac function associated to a better antioxidant response and lower type 3 iodothyronine deiodinase activity after myocardial infarction. PLOS ONE 14, e0222334 (2019).

11. Stolen, T. et al. Effect of exercise training on cardiac metabolism in rats with heart failure. Scand. Cardiovasc. J. https://doi.org/10.1080/14017431.2019.1658893 (2019).

12. Taylor, R. S. et al. Impact of exercise rehabilitation on exercise capacity and quality-of-life in heart failure: individual participant meta-analysis. J. Am. Coll. Cardiol. 73, 1430-1443 (2019).

13. Ding, R. Exercise-based rehabilitation for heart failure: clinical evidence. Adv. Exp. Med. Biol. 1000, 31-49 (2017).

14. Luan, X. et al. Exercise as a prescription for patients with various diseases. J. Sport Health Sci. 8, 422-441 (2019).

15. Lavie, C. J. et al. Exercise and the cardiovascular system: clinical science and cardiovascular outcomes. Circ. Res. 117, 207-219 (2015).

16. Wang, L., Wang, J., Cretoiu, D., Li, G. \& Xiao, J. Exercise-mediated regulation of autophagy in the cardiovascular system. J. Sport Health Sci. 9, 203-210 (2020).

17. Cattadori, G., Segurini, C., Picozzi, A., Padeletti, L. \& Anza, C. Exercise and heart failure: an update. ESC Heart Fail 5, 222-232 (2018). 
18. Schachinger, V., Britten, M. B. \& Zeiher, A. M. Prognostic impact of coronary vasodilator dysfunction on adverse long-term outcome of coronary heart disease. Circulation 101, 1899-1906 (2000).

19. Aengevaeren, V. L. et al. Exercise-induced cardiac troponin I increase and incident mortality and cardiovascular events. Circulation 140, 804-814 (2019).

20. Sanchis-Gomar, F., Perez, L. M., Joyner, M. J., Lollgen, H. \& Lucia, A. Endurance exercise and the heart: friend or foe? Sports Med. 46, 459-466 (2016).

21. Mediano, M. F. F. et al. Influence of baseline physical activity level on exercise training response and clinical outcomes in heart failure: the HF-ACTION trial. JACC Heart Fail 6, 1011-1019 (2018).

22. Barboza, C. A. et al. Cardioprotective properties of aerobic and resistance training against myocardial infarction. Int. J. Sports Med. 37, 421-430 (2016).

23. Liu, X. et al. miR-222 is necessary for exercise-induced cardiac growth and protects against pathological cardiac remodeling. Cell Metab. 21, 584-595 (2015).

24. Hackam, D. G. et al. The 2010 Canadian Hypertension Education Program recommendations for the management of hypertension: part 2 - therapy. Can. J. Cardiol. 26, 249-258 (2010).

25. Franzoni, F. et al. Physical activity, plasma antioxidant capacity, and endothelium-dependent vasodilation in young and older men. Am. J. Hypertens. 18, 510-516 (2005).

26. Korsager Larsen, M. \& Matchkov, V. V. Hypertension and physical exercise: the role of oxidative stress. Medicine 52, 19-27 (2016).

27. Kodama, S. et al. Fasting and post-challenge glucose as quantitative cardiovascular risk factors: a meta-analysis. J. Atheroscler. Thromb. 19, 385-396 (2012).

28. Kim, D. I. et al. Six weeks of combined aerobic and resistance exercise using outdoor exercise machines improves fitness, insulin resistance, and chemerin in the Korean elderly: A pilot randomized controlled trial. Arch. Gerontol. Geriatr. 75, 59-64 (2018).

29. Hur, S., Cho, S. H., Song, B. K. \& Cho, B. J. Effect of resistance exercise on serum osteoprotegerin levels and insulin resistance in middle-aged women with metabolic syndrome. Med Sci. Monit. 24, 9385-9391 (2018).

30. Hannemann, J. et al. Timed physical exercise does not influence circadian rhythms and glucose tolerance in rotating night shift workers: The EuRhythDia study. Diab Vasc. Dis. Res. 17, 1479164120950616 (2020).

31. Marzuca-Nassr, G. N. et al. High-intensity interval training on body composition, functional capacity and biochemical markers in healthy young versus older people. Exp. Gerontol. 141, 111096 (2020).

32. Nery, C. et al. Effectiveness of resistance exercise compared to aerobic exercise without insulin therapy in patients with type 2 diabetes mellitus: a metaanalysis. Braz. J. Phys. Ther. 21, 400-415 (2017).

33. Lee, S. et al. Effects of exercise modality on insulin resistance and ectopic fat in adolescents with overweight and obesity: a randomized clinical trial. J. Pediatr. 206, 91-98 (2019)

34. Abdelbasset, W. K. Resistance exercise versus aerobic exercise combined with metformin therapy in the treatment of type 2 diabetes: a 12-week comparative clinical study. Endocr. Metab. Immune Disord. Drug Targets https://doi.org/ 10.2174/1871530320999200918143227 (2020)

35. Pierce, J. R. et al. Growth hormone and insulin-like growth factor-I molecular weight isoform responses to resistance exercise are sex-dependent. Front. Endocrinol. 11, 571 (2020).

36. Hougham, K. A. \& Ross, R. Evidence that the association between exercise intensity and insulin sensitivity is sex dependent. Appl. Physiol. Nutr. Metab. 36, 730-735 (2011).

37. Nakamura, M. \& Sadoshima, J. Mechanisms of physiological and pathological cardiac hypertrophy. Nat. Rev. Cardiol. 15, 387-407 (2018).

38. Schuttler, D., Clauss, S., Weckbach, L. T. \& Brunner, S. Molecular mechanisms of cardiac remodeling and regeneration in physical exercise. Cells https://doi.org/ 10.3390/cells8101128 (2019).

39. Conti, V. et al. Aerobic training workload affects human endothelial cells redox homeostasis. Med. Sci. Sports Exerc. 45, 644-653 (2013).

40. Brahmer, A. et al. Platelets, endothelial cells and leukocytes contribute to the exercise-triggered release of extracellular vesicles into the circulation. J. Extracell. Vesicles 8, 1615820 (2019).

41. Xia, W. H. et al. Physical exercise attenuates age-associated reduction in endothelium-reparative capacity of endothelial progenitor cells by increasing CXCR4/JAK-2 signaling in healthy men. Aging Cell 11, 111-119 (2012).

42. Varga, I., Kyselovic, J., Galfiova, P. \& Danisovic, L. The non-cardiomyocyte cells of the heart. Their possible roles in exercise-induced cardiac regeneration and remodeling. Adv. Exp. Med. Biol. 999, 117-136 (2017).

43. Wakatsuki, T., Schlessinger, J. \& Elson, E. L. The biochemical response of the heart to hypertension and exercise. Trends Biochem. Sci. 29, 609-617 (2004).

44. Lighthouse, J. K. et al. Exercise promotes a cardioprotective gene program in resident cardiac fibroblasts. $J C l$ Insight https://doi.org/10.1172/jci.insight.92098 (2019).
45. Peake, J. M., Neubauer, O., Walsh, N. P. \& Simpson, R. J. Recovery of the immune system after exercise. J. Appl. Physiol. 122, 1077-1087 (2017).

46. Weeks, K. L., Bernardo, B. C., Ooi, J. Y. Y., Patterson, N. L. \& McMullen, J. R. The IGF1-PI3K-Akt signaling pathway in mediating exercise-induced cardiac hypertrophy and protection. Adv. Exp. Med. Biol. 1000, 187-210 (2017).

47. Essandoh, K. et al. Tsg101 positively regulates physiologic-like cardiac hypertrophy through FIP3-mediated endosomal recycling of IGF-1R. FASEB J. 33, 7451-7466 (2019).

48. DeBosch, B. et al. Akt1 is required for physiological cardiac growth. Circulation 113, 2097-2104 (2006).

49. Shioi, T. et al. Akt/protein kinase B promotes organ growth in transgenic mice. Mol. Cell Biol. 22, 2799-2809 (2002).

50. Bostrom, P. et al. C/EBPbeta controls exercise-induced cardiac growth and protects against pathological cardiac remodeling. Cell 143, 1072-1083 (2010).

51. Bezzerides, V. J. et al. CITED4 induces physiologic hypertrophy and promotes functional recovery after ischemic injury. $J C l$ Insight https://doi.org/10.1172/jci. insight.85904 (2016)

52. Ryall, K. A., Bezzerides, V. J., Rosenzweig, A. \& Saucerman, J. J. Phenotypic screen quantifying differential regulation of cardiac myocyte hypertrophy identifies CITED4 regulation of myocyte elongation. J. Mol. Cell Cardiol. 72, 74-84 (2014).

53. Oka, T. et al. Cardiac-specific deletion of Gata4 reveals its requirement for hypertrophy, compensation, and myocyte viability. Circ. Res. 98, 837-845 (2006).

54. Naderi, N. et al. High-intensity interval training increase GATA4, CITED4 and c-Kit and decreases C/EBPbeta in rats after myocardial infarction. Life Sci. 221, 319-326 (2019).

55. Wang, L., Lv, Y., Li, G. \& Xiao, J. MicroRNAs in heart and circulation during physical exercise. J. Sport Health Sci. 7, 433-441 (2018).

56. Wu, G., Zhang, X. \& Gao, F. The epigenetic landscape of exercise in cardiac health and disease. J. Sport Health Sci. https://doi.org/10.1016/j.jshs.2020.12.003 (2020).

57. Shi, J. et al. miR-17-3p contributes to exercise-induced cardiac growth and protects against myocardial ischemia-reperfusion injury. Theranostics 7, 664-676 (2017).

58. Chen, Z. et al. miR-17 regulates the proliferation and apoptosis of endothelial cells in coronary heart disease via targeting insulin-like-growth factor 1. Pathol. Res. Pract. 215, 152512 (2019).

59. Chen, J. et al. mir-17-92 cluster is required for and sufficient to induce cardiomyocyte proliferation in postnatal and adult hearts. Circ. Res. 112, 1557-1566 (2013).

60. Kasinski, A. L. \& Slack, F. J. Epigenetics and genetics. MicroRNAs en route to the clinic: progress in validating and targeting microRNAs for cancer therapy. Nat. Rev. Cancer 11, 849-864 (2011).

61. Colombo, M., Raposo, G. \& Thery, C. Biogenesis, secretion, and intercellular interactions of exosomes and other extracellular vesicles. Annu. Rev. Cell Dev. Biol. 30, 255-289 (2014).

62. Thery, C., Amigorena, S., Raposo, G. \& Clayton, A. Isolation and characterization of exosomes from cell culture supernatants and biological fluids. Curr. Protoc. Cell Biol. https://doi.org/10.1002/0471143030.cb0322s30 (2006).

63. Mathivanan, S., Ji, H. \& Simpson, R. J. Exosomes: extracellular organelles important in intercellular communication. J. Proteomics 73, 1907-1920 (2010).

64. Hou, Z. et al. Longterm exercise-derived exosomal miR-342-5p. Circ. Res. 124, 1386-1400 (2019)

65. D'Souza, R. F. et al. Circulatory exosomal miRNA following intense exercise is unrelated to muscle and plasma miRNA abundances. Am. J. Physiol. Endocrinol. Metab. 315, E723-E733 (2018).

66. Liao, Z. Y. et al. The effect of exercise, resveratrol or their combination on Sarcopenia in aged rats via regulation of AMPK/Sirt1 pathway. Exp. Gerontol. 98, 177-183 (2017).

67. Jia, D., Hou, L., Lv, Y., Xi, L. \& Tian, Z. Postinfarction exercise training alleviates cardiac dysfunction and adverse remodeling via mitochondrial biogenesis and SIRT1/PGC-1alpha/PI3K/Akt signaling. J. Cell Physiol. 234, 23705-23718 (2019).

68. Lai, C. H. et al. Exercise training enhanced SIRT1 longevity signaling replaces the IGF1 survival pathway to attenuate aging-induced rat heart apoptosis. Age $\mathbf{3 6}$, 9706 (2014).

69. Packer, M. Longevity genes, cardiac ageing, and the pathogenesis of cardiomyopathy: implications for understanding the effects of current and future treatments for heart failure. Eur. Heart J. https://doi.org/10.1093/eurheartj/ ehaa360 (2020)

70. Asokan, S. M., Wang, T., Wang, M. F. \& Lin, W. T. A novel dipeptide from potato protein hydrolysate augments the effects of exercise training against high-fat diet-induced damages in senescence-accelerated mouse-prone 8 by boosting pAMPK/SIRT1/PGC-1alpha/pFOXO3 pathway. Aging 12, 7334-7349 (2020).

71. Prola, A. et al. SIRT1 protects the heart from ER stress-induced cell death through elF2alpha deacetylation. Cell Death Differ. 24, 343-356 (2017). 
72. Li, X. Y., Han, X., Zhang, H. M., Tan, H. \& Han, S. F. [SIRT1 signaling pathway mediated the protective effects on myocardium of rats after endurance training and acute exhaustive exercise]. Zhonghua Xin Xue Guan Bing. Za Zhi 45, 501-506 (2017).

73. Copple, I. M. The Keap1-Nrf2 cell defense pathway-a promising therapeutic target? Adv. Pharmācol. 63, 43-79 (2012).

74. Done, A. J. \& Traustadottir, T. Nrf2 mediates redox adaptations to exercise. Redox Biol. 10, 191-199 (2016).

75. Done, A. J., Gage, M. J., Nieto, N. C. \& Traustadottir, T. Exercise-induced Nrf2signaling is impaired in aging. Free Radic. Biol. Med. 96, 130-138 (2016).

76. Stewart, K. J., Ouyang, P., Bacher, A. C., Lima, S. \& Shapiro, E. P. Exercise effects on cardiac size and left ventricular diastolic function: relationships to changes in fitness, fatness, blood pressure and insulin resistance. Heart 92, 893-898 (2006).

77. Yalcin, F. et al. Evolution of ventricular hypertrophy and myocardial mechanics in physiological and pathological hypertrophy. J. Appl Physiol. 126, 354-362 (2019).

78. Lerchenmuller, C. \& Rosenzweig, A. Mechanisms of exercise-induced cardiac growth. Drug Discov. Today 19, 1003-1009 (2014).

79. Dunn, M. E. et al. Serum natriuretic peptides as differential biomarkers allowing for the distinction between physiologic and pathologic left ventricular hypertrophy. Toxicol. Pathol. 45, 344-352 (2017).

80. Jamshidi, Y. et al. Peroxisome proliferator-activated receptor alpha gene regulates left ventricular growth in response to exercise and hypertension. Circulation 105, 950-955 (2002).

81. Neri Serneri, G. G. et al. Increased cardiac sympathetic activity and insulin-like growth factor-I formation are associated with physiological hypertrophy in athletes. Circ. Res. 89, 977-982 (2001).

82. Weeks, K. L. et al. Phosphoinositide 3-kinase p110alpha is a master regulator of exercise-induced cardioprotection and PI3K gene therapy rescues cardiac dysfunction. Circ. Heart Fail 5, 523-534 (2012).

83. Lee, H. W. et al. Effects of exercise training and TrkB blockade on cardiac function and BDNF-TrkB signaling postmyocardial infarction in rats. Am. J. Physiol. Heart Circ. Physiol. 315, H1821-H1834 (2018).

84. Trivedi, C. M. \& Epstein, J. A. Heart-healthy hypertrophy. Cell Metab. 13, 3-4 (2011).

85. Zou, J. et al. C/EBPbeta knockdown protects cardiomyocytes from hypertrophy via inhibition of p65-NFkappaB. Mol. Cell Endocrinol. 390, 18-25 (2014).

86. Liao, Z. et al. Early moderate exercise benefits myocardial infarction healing via improvement of inflammation and ventricular remodelling in rats. J. Cell Mol. Med. https://doi.org/10.1111/jcmm.14710 (2019).

87. McNamara, D. A. et al. Left atrial electromechanical remodeling following 2 years of high-intensity exercise training in sedentary middle-aged adults. Circulation 139, 1507-1516 (2019).

88. Zhang, Y. M. et al. The effects of different initiation time of exercise training on left ventricular remodeling and cardiopulmonary rehabilitation in patients with left ventricular dysfunction after myocardial infarction. Disabil. Rehabil. 38, 268-276 (2016).

89. La Gerche, A. et al. Disproportionate exercise load and remodeling of the athlete's right ventricle. Med. Sci. Sports Exerc. 43, 974-981 (2011).

90. Aengevaeren, V. L. et al. Right heart remodeling in olympic athletes during 8 years of intensive exercise training. J. Am. Coll. Cardiol. 72, 815-817 (2018).

91. Calvert, J. W. et al. Exercise protects against myocardial ischemia-reperfusion injury via stimulation of beta(3)-adrenergic receptors and increased nitric oxide signaling: role of nitrite and nitrosothiols. Circ. Res. 108, 1448-1458 (2011).

92. Li, J. et al. Alpha-catenins control cardiomyocyte proliferation by regulating Yap activity. Circ. Res. 116, 70-79 (2015).

93. Bergmann, O. et al. Evidence for cardiomyocyte renewal in humans. Science 324, 98-102 (2009).

94. Lazar, E., Sadek, H. A. \& Bergmann, O. Cardiomyocyte renewal in the human heart: insights from the fall-out. Eur. Heart J. 38, 2333-2342 (2017).

95. Vujic, A. et al. Exercise induces new cardiomyocyte generation in the adult mammalian heart. Nat. Commun. 9, 1659 (2018).

96. Huang, G. N. et al. C/EBP transcription factors mediate epicardial activation during heart development and injury. Science 338, 1599-1603 (2012).

97. Ding, S. et al. C/EBPB-CITED4 in exercised heart. Adv. Exp. Med. Biol. 1000, 247-259 (2017).

98. Asif, Y. et al. Sustained cardiac programming by short-term juvenile exercise training in male rats. J. Physiol. 596, 163-180 (2018).

99. Poss, K. D., Wilson, L. G. \& Keating, M. T. Heart regeneration in zebrafish. Science 298, 2188-2190 (2002).

100. Rovira, M., Borras, D. M., Marques, I. J., Puig, C. \& Planas, J. V. Physiological responses to swimming-induced exercise in the adult zebrafish regenerating heart. Front. Physiol. 9, 1362 (2018).

101. Narula, J. et al. Apoptosis in myocytes in end-stage heart failure. N. Engl. J. Med. 335, 1182-1189 (1996)
102. Foo, R. S., Mani, K. \& Kitsis, R. N. Death begets failure in the heart. J. Clin. Invest. 115, 565-571 (2005).

103. Parmacek, M. S. \& Epstein, J. A. Cardiomyocyte renewal. N. Engl. J. Med. 361, 86-88 (2009).

104. Kiuchi, S. et al. Cardiac pacemaker cells generate cardiomyocytes from fibroblasts in long-term cultures. Sci. Rep. 9, 15174 (2019).

105. Olivetti, G. et al. Apoptosis in the failing human heart. N. Engl. J. Med. 336, 1131-1141 (1997).

106. Cheedipudi, S. M. et al. Exercise restores dysregulated gene expression in a mouse model of arrhythmogenic cardiomyopathy. Cardiovasc. Res. https://doi. org/10.1093/cvr/cvz199 (2019).

107. Siu, P. M., Bryner, R. W., Martyn, J. K. \& Alway, S. E. Apoptotic adaptations from exercise training in skeletal and cardiac muscles. FASEB J. 18, 1150-1152 (2004).

108. Verjans, R. et al. MicroRNA-221/222 family counteracts myocardial fibrosis in pressure overload-induced heart failure. Hypertension 71, 280-288 (2018).

109. Werner, C. et al. Effects of physical exercise on myocardial telomere-regulating proteins, survival pathways, and apoptosis. J. Am. Coll. Cardiol. 52, 470-482 (2008).

110. Ludlow, A. T., Gratidao, L., Ludlow, L. W., Spangenburg, E. E. \& Roth, S. M. Acute exercise activates p38 MAPK and increases the expression of telomereprotective genes in cardiac muscle. Exp. Physiol. 102, 397-410 (2017).

111. Stellos, K. \& Spyridopoulos, I. Exercise, telomerase activity, and cardiovascular disease prevention. Eur. Heart J. 40, 47-49 (2019).

112. Lee, Y., Kwon, I., Jang, Y., Cosio-Lima, L. \& Barrington, P. Endurance exercise attenuates doxorubicin-induced cardiotoxicity. Med. Sci. Sports Exerc. https://doi. org/10.1249/MSS.0000000000002094 (2019).

113. Hambrecht, R. et al. Regular physical exercise corrects endothelial dysfunction and improves exercise capacity in patients with chronic heart failure. Circulation 98, 2709-2715 (1998).

114. Teixeira, M. et al. Regular exercise participation contributes to better proteostasis, inflammatory and vasoactive profiles in patients with hypertension. Am. J. Hypertens. https://doi.org/10.1093/ajh/hpz160 (2019).

115. Moncada, S., Palmer, R. M. \& Higgs, E. A. Nitric oxide: physiology, pathophysiology, and pharmacology. Pharmacol. Rev. 43, 109-142 (1991).

116. Vallance, P., Collier, J. \& Moncada, S. Effects of endothelium-derived nitric oxide on peripheral arteriolar tone in man. Lancet 2, 997-1000 (1989).

117. Sessa, W. C., Pritchard, K., Seyedi, N., Wang, J. \& Hintze, T. H. Chronic exercise in dogs increases coronary vascular nitric oxide production and endothelial cell nitric oxide synthase gene expression. Circ. Res. 74, 349-353 (1994).

118. Griffin, K. L., Mattox, M. L., Larkin, B. D., Laughlin, M. H. \& Parker, J. L. Exercise training enhances endothelium-dependent relaxation of porcine coronary arteries distal to chronic coronary occlusion. Shock 11, 56-56 (1999).

119. Wang, Y. X. et al. ROS and NO dynamics in endothelial cells exposed to exerciseinduced wall shear stress. Cell Mol. Bioeng. 12, 107-120 (2019).

120. Domanska-Senderowska, D. et al. MicroRNA profile and adaptive response to exercise training: a review. Int. J. Sports Med. 40, 227-235 (2019).

121. Cai, Y., Xie, K. L., Zheng, F. \& Liu, S. X. Aerobic exercise prevents insulin resistance through the regulation of miR-492/resistin axis in aortic endothelium. J. Cardiovasc. Transl. Res. 11, 450-458 (2018).

122. Donghui, T. et al. Improvement of microvascular endothelial dysfunction induced by exercise and diet is associated with microRNA-126 in obese adolescents. Microvasc. Res. 123, 86-91 (2019).

123. Li, Y., Zhang, H. \& Wang, Y. Tai Chi ameliorates coronary heart disease by affecting serum levels of miR-24 and miR-155. Front. Physiol. 10, 587 (2019).

124. Davis, J., Burr, A. R., Davis, G. F., Birnbaumer, L. \& Molkentin, J. D. A TRPC6dependent pathway for myofibroblast transdifferentiation and wound healing in vivo. Dev. Cell 23, 705-715 (2012).

125. Fernandes, T., Barauna, V. G., Negrao, C. E., Phillips, M. I. \& Oliveira, E. M. Aerobic exercise training promotes physiological cardiac remodeling involving a set of microRNAs. Am. J. Physiol. Heart Circ. Physiol. 309, H543-H552 (2015).

126. Dawson, K. et al. MicroRNA29: a mechanistic contributor and potential biomarker in atrial fibrillation. Circulation 127, 1466-1475 (2013).

127. Brianezi, L. et al. Effects of physical training on the myocardium of oxariectomized LDLr knockout mice: MMP 2/9, collagen I/III, inflammation and oxidative stress. Arq. Bras. Cardiol. https://doi.org/10.5935/abc.20190223 (2019).

128. Nogueira-Ferreira, R. et al. One year of exercise training promotes distinct adaptations in right and left ventricle of female Sprague-Dawley rats. J. Physiol. Biochem. https://doi.org/10.1007/s13105-019-00705-4 (2019).

129. Raposo, G. \& Stoorvogel, W. Extracellular vesicles: exosomes, microvesicles, and friends. J. Cell Biol. 200, 373-383 (2013).

130. Farooqi, A. A. et al. Exosome biogenesis, bioactivities and functions as new delivery systems of natural compounds. Biotechnol. Adv. 36, 328-334 (2018).

131. Bei, Y. et al. Exercise-induced circulating extracellular vesicles protect against cardiac ischemia-reperfusion injury. Basic Res. Cardiol. 112, 38 (2017). 
132. Li, Y. et al. Exosomes mediate the beneficial effects of exercise. Adv. Exp. Med. Biol. 1000, 333-353 (2017).

133. Fruhbeis, C., Helmig, S., Tug, S., Simon, P. \& Kramer-Albers, E. M. Physical exercise induces rapid release of small extracellular vesicles into the circulation. $J$. Extracell. Vesicles 4, 28239 (2015).

134. Chaturvedi, P., Kalani, A., Medina, I., Familtseva, A. \& Tyagi, S. C. Cardiosome mediated regulation of MMP9 in diabetic heart: role of mir29b and mir455 in exercise. J. Cell Mol. Med. 19, 2153-2161 (2015).

135. Wang, X. et al. Cardiomyocytes mediate anti-angiogenesis in type 2 diabetic rats through the exosomal transfer of miR-320 into endothelial cells. J. Mol. Cell Cardiol. 74, 139-150 (2014).

136. Wahl, P. et al. Acute effects of different exercise protocols on the circulating vascular microRNAs -16, -21, and -126 in trained subjects. Front. Physiol. 7, 643 (2016).

137. $\mathrm{Ma}, \mathrm{C}$. et al. Moderate exercise enhances endothelial progenitor cell exosomes release and function. Med. Sci. Sports Exerc. 50, 2024-2032 (2018).

138. Ghorbanzadeh, V. et al. Cardioprotective effect of crocin combined with voluntary exercise in rat: role of Mir-126 and Mir-210 in heart angiogenesis. Arq. Bras. Cardiol. 109, 54-62 (2017).

139. Whitham, M. et al. Extracellular vesicles provide a means for tissue crosstalk during exercise. Cell Metab. 27, 237-251 (2018). e234.

140. Wang, Y. et al. Six-year incidence of cardiometabolic risk factors in a populationbased cohort of Chinese adults followed from 2009 to 2015. J. Am. Heart Assoc 8, e011368 (2019).

141. Motahari-Tabari, N., Ahmad Shirvani, M., Shirzad, E. A. M., Yousefi-Abdolmaleki, E. \& Teimourzadeh, M. The effect of 8 weeks aerobic exercise on insulin resistance in type 2 diabetes: a randomized clinical trial. Glob. J. Health Sci. 7, 115-121 (2014).

142. Ho, S. S., Dhaliwal, S. S., Hills, A. P. \& Pal, S. The effect of 12 weeks of aerobic, resistance or combination exercise training on cardiovascular risk factors in the overweight and obese in a randomized trial. BMC Public Health 12, 704 (2012).

143. Marc-Hernandez, A., Ruiz-Tovar, J., Aracil, A., Guillen, S. \& Moya-Ramon, M. Impact of exercise on body composition and cardiometabolic risk factors in patients awaiting bariatric surgery. Obes. Surg. 29, 3891-3900 (2019).

144. Lin, X. et al. Effects of exercise training on cardiorespiratory fitness and biomarkers of cardiometabolic health: a systematic review and meta-analysis of randomized controlled trials. J. Am. Heart Assoc. https://doi.org/10.1161/ JAHA.115.002014 (2015).

145. Laker, R. C. et al. Transcriptomic and epigenetic responses to short-term nutrient-exercise stress in humans. Sci. Rep. 7, 15134 (2017).

146. Hu, Z., Zhou, L. \& He, T. Potential effect of exercise in ameliorating insulin resistance at transcriptome level. J. Sports Med. Phys. Fit. 59, 116-125 (2019).

147. Niu, Y. et al. Exercise-induced GLUT4 transcription via inactivation of HDAC4/5 in mouse skeletal muscle in an AMPKalpha2-dependent manner. Biochim. Biophys. Acta Mol. Basis Dis. 1863, 2372-2381 (2017).

148. Gianotti, T. F. et al. A decreased mitochondrial DNA content is related to insulin resistance in adolescents. Obesity (Silver Spring) 16, 1591-1595 (2008).

149. Santos, J. L. et al. Plasma lactate and leukocyte mitochondrial DNA copy number as biomarkers of insulin sensitivity in non-diabetic women. J. Physiol. Biochem. 75, 285-297 (2019).

150. Benite-Ribeiro, S. A., Lucas-Lima, K. L., Jones, J. N. \& Dos Santos, J. M. Transcription of mtDNA and dyslipidemia are ameliorated by aerobic exercise in type 2 diabetes. Mol. Biol. Rep. https://doi.org/10.1007/s11033-020-05725-8 (2020).

\section{ACKNOWLEDGEMENTS}

This work was supported by the grants from National Key Research and Development Project (2018YFE0113500 to J.J.X.), National Natural Science Foundation of China (82020108002 and 81911540486 to J.J.X.), Innovation Program of Shanghai Municipal Education Commission (2017-01-07-00-09-E00042 to J.J.X.), the grant from Science and Technology Commission of Shanghai Municipality (20DZ2255400 and 18410722200 to J.J.X.), the "Dawn" Program of Shanghai Education Commission (19SG34 to J.J.X.), the Sailing Program from Science and Technology Commission of Shanghai (20YF1414000 to H.Y.W.), "Chenguang Program" of Shanghai Education Development Foundation and Shanghai Municipal Education Commission (20CG46 to H.Y.W.) and National Natural Science Foundation of China (82000253 to H.Y.W.).

\section{AUTHOR CONTRIBUTIONS}

J.X. had the idea for the article. H.W. and Y.X. performed the literature search and analysis. H.W., L.G., K.E., and J.X. drafted and critically revised the work.

\section{COMPETING INTERESTS}

The authors declare no competing interests.

\section{ETHICS APPROVAL}

This article does not contain any studies with human participants and animals performed by any of the authors.

\section{ADDITIONAL INFORMATION}

Correspondence and requests for materials should be addressed to J.X.

Reprints and permission information is available at http://www.nature.com/ reprints

Publisher's note Springer Nature remains neutral with regard to jurisdictional claims in published maps and institutional affiliations.

Open Access This article is licensed under a Creative Commons Attribution 4.0 International License, which permits use, sharing, adaptation, distribution and reproduction in any medium or format, as long as you give appropriate credit to the original author(s) and the source, provide a link to the Creative Commons license, and indicate if changes were made. The images or other third party material in this article are included in the article's Creative Commons license, unless indicated otherwise in a credit line to the material. If material is not included in the article's Creative Commons license and your intended use is not permitted by statutory regulation or exceeds the permitted use, you will need to obtain permission directly from the copyright holder. To view a copy of this license, visit http://creativecommons. org/licenses/by/4.0/.

(c) The Author(s) 2021 